\title{
Light frames the shadow: surgical teaching and education in 2015
}

\author{
F. M. Riegler
}

Published online: 10 April 2015

(C) Springer-Verlag Wien 2015

\section{Dear reader,}

Within the continuous process of acquisition of knowledge, the academic life aims to outbalance teaching, education, research, and clinical practice. Academic politics serve to provide the necessary logistic platform and organization to meet the requirements of the modern university. As both science and politics are orchestrated by the large diametral spectrum of personalities involved, reasoning, and definition of success is largely colored by human being characteristics of so-called egodriven social behavior. These wonderful spectra of the rainbow experience involve self-esteem, power gain goiter game googling gambling, greed need feed, soil seed sampler, and hate harbors humanity. All wants more. Therefore, it is all as we make it: positive and/or negative. Sky moves the clouds. Sun shines, reflection shades. Here we clearly sense the positive art of medicine, the positive art of diagnosis and therapy, the positive art of surgery, the positive art for sensing the essence of a disease, and considering the essence the modern artist designs the management of disease.

Let me draw your attention on the present issue of European Surgery. Going in line with the fascinating modern developments this issue of European Surgery draws YOUR attention on an outstanding aspect of novel medicine. The team around the well-known and highly reputed Prof. Reinhold Kafka-Ritsch from the Medical University Innsbruck, Tyrol, Austria composed a fascinating symphony of articles on modern approach in surgery [1-6]. Including novel technologies, surgical techniques and treatment modalities the concept of fast track sur-

F. M. Riegler $(\square)$

Reflux Medical,

Mariannengasse 10/9,

1090 Vienna, Austria

e-mail: martin.riegler@refluxmedical.com gery is outlined and well presented. I hope that you also share my enthusiasm for the articles [1-6]. In addition this issue contains articles on special topics on laparoscopic and open colorectal surgery $[7,8]$. Remains to be questioned if the understanding of the essence of a given disease may foster the development of better treatment?

Diagnosis and treatment aim to elucidate the reason for the impairment of life quality and productivity: pain and/or cancer risk, that is, stress. Consequently the modern approach includes the following stress factors, which in turn may impair the stability of a given organism: physical stress (bad shoes); inflammatory/oncologic stress (IBD, You BD, NERD-Milano classes, etc.); metabolic stress (sugar minot rubber dub disaster of diabetes); emotional stress (every need got an ego to feed). Essence directed diagnosis aims to define and characterize the cause(s) of stress to develop a tailored therapy. Elimination of impaired life quality and productivity defines success. This conduct of reasoning should be the basis of the modern medical university. As a matter of fact this can only be achieved by a multidisciplinary open minded approach, which in turn incorporates teaching, education, clinical practice, and skills. Finally academic politics must offer the important economic and platform resources to foster the independence of the academy. Conceptually time space and love are the foundations for the expression of essence directed modern medicine. Watch out and let it be done. Fuse the blues and let it reggae to sun the shine in the positive way of vibrations. At the end of the day you will have happy academic ancestors for the future generations of academic medical science and clinical practice. Reduce the impact of hate, greed, envy and self-esteem compensation. Exodus via the Pessach jam suff red sea experience to the promised land stimulates to get individual freedom and harmony $[9,10]$. Jamming follows Exodus [9, 10] and represents an order of deeper semiotic meaning. Outbalance the powers to grow the beautiful flowers of the future. Reveal the 


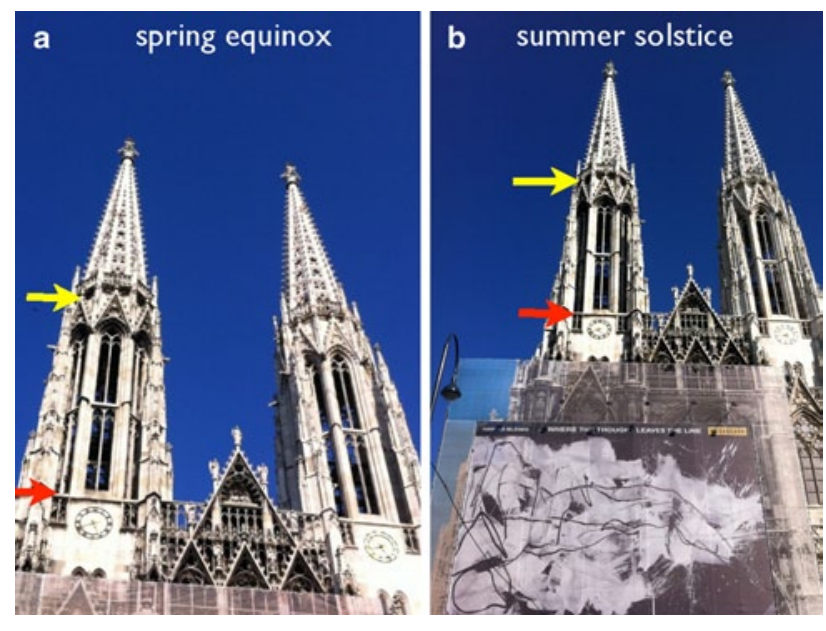

Fig. 1 Season dependent sun shadow effect on the Vienna votive church twin towers window openings. a At spring equinox sunrise the window opening extending between the yellow and red arrow only partially includes a shadow within the upper portion of the window opening. b At summer solstice the window opening between the yellow and red arrow includes a shadow, thus completely eradicating the window opening and bringing it out of the perception. Images obtained by the author during sunrise at spring equinox 2014 (21.03.2015) and summer solstice 2014 (21.06.2014), using iPhone technology. Image mirrors the idea, that architecture aims to serve as cosmic clock, as described in the text

remembrance for the soil and the roots. Stop to poison the minds of the employees and foster the outbalance of teaching and education [11]. Uprising of the new time will bring back the scholar following the teacher with honor, respect, and adoration. Otherwise the excel list shaped towers will become the prison of the morals, which solely serve to justify the power games of those involved. It all will continue to chill off in the form of senseless calculations, statistics, and logistic regressions. Talk to each other and open your minds against the deficit of respect, attention, and humility. Sun rises to put light on the golden center of excellence.

Perception of architecture becomes art if the underlying semiotic meaning is well understood [11]. Thus, for example, buildings are orientated along the axis of the solar spring equinoxe and thus represent a mirror of the cosmos [11]. Space is time and mass increases with speed. The velocity of light incorporates the largest amount of mass. Relativity teaches that mass influences the direction of light. Going in line with this concept, let us catch a smile from the towers of the votive church in Vienna, Austria, Europe. Originally the church has been erected as a sign of gratitude for the salvation of the Emperor Franz Joseph I from being killed during an hostile attack 1853. The front twin towers of the church harbor large window openings (Fig. 1). Depending on the position of the sun during sunrise at spring equinox (Fig. la) or summer solstice (Fig. 1b), the shadows partially (Fig. 1a) or completely (Fig. 1b) fill the large window openings of the front twin towers of the church. As a consequence, the windows seem to disappear at the summer solstice (Fig. 1b). Light frames the shadow. Here architecture unhides the deep meaning of a cosmic clock, which connects those passing by with the huge scale of the universe. One gets touched by an intense flash of remembrance for the "physis kryptestai philei", by Heraclitus. This statement indicates, that all things that we perceive love, tend and intend to hide their essence. Thus adoration and gratitude for being should motivate our actions, at least as surgeons and readers of European Surgery. Stay tuned, withstand the emotional tides of the current political developments, watch your step and enjoy YOUR day.

Martin Riegler.

\section{Conflict of interest}

The author declares that there exists no conflict of interest.

\section{References}

1. Kafka-Ritsch R. Is fast track rehabilitation already standard in gastrointestinal surgery? Eur Surg. 2015;47:42-5.

2. Bräunlein J, Gasser E, Kafka-Ritsch R. Compliance with and fulfillment of a fast-track protocol in daily surgical practice in a university hospital. Eur Surg. 2015;47:46-9.

3. Möschel M, Wohlgenannt D. Is epidural anaesthesia mandatory in fast-track surgery for elective colorectal resections? Eur Surg. 2015;47:50-3.

4. Anschütz M, Drautz J, Kafka-Ritsch R. Effect of perioperative epidural anesthesia in elective laparoscopic colorectal resections. Eur Surg. 2015;47:54-6.

5. Drautz J, Anschütz M, Kafka-Ritsch R. Amount and influence of perioperative fluid administration on outcome in routine elective colorectal surgery according to an ERAS protocol. Eur Surg. 2015;47:57-9.

6. Zacherl J, Asari R, Fleischmann E, et al. Fast-track Ivor Lewis esophageal resection. Eur Surg. 2015;47:60-5.

7. Dulskas A, Samalavicius NE, Gupta RK, et al. Functional and clinical outcomes of hand-assisted laparoscopic colorectal surgery: a single-institution experience in 255 patients. Eur Surg. 2015;47:76-81.

8. Pellino G, Selvaggi F. From colon-sparing techniques to pelvic ileal pouch: history and evolution of surgery for ulcerative colitis. Eur Surg. 2015;47:82-91.

9. Marley B. Exodus. Bob Marley and The Wailers. Island Records 1977.

10. Marley B. Jamming (= Jam Suff). Bob Marley and The Wailers. Island Records 1977.

11. Riegler FM. For the beauty of our globe. Eur Surg. 2015;47:1-8. 